\title{
Comparación del desarrollo motor en escolares de 9 y 10 años de edad en clases de educación física y talleres deportivos extracurriculares
}

\author{
Comparison of motor development in school aged children ages 9 and 10 in physi- \\ cal education classes and extracurricular sport workshops
}

\author{
*Ximena Pradenas Vargas; *María Campos García; *Matias Contreras Sepúlveda; \\ *Daniela Puentes Matus; *Pablo Luna Villouta
}

Pradenas, X.; Campos, M.; Contreras, M.; Puentes,D. \& Luna, P. (2017). Comparación del desarrollo motor en escolares de 9 y 10 años de edad en clases de educación física y talleres deportivos extracurriculares. Revista Ciencias de la Actividad Física UCM, No 18(2) julio-diciembre, 1-8.

\section{RESUMEN}

La investigación buscó determinar si existen diferencias en el desarrollo motor en escolares de 9 y 10 años, que participan únicamente de la clase de educación física, y aquellos que además de la clase, participan de talleres deportivos extracurriculares pertenecientes a colegios particulares subvencionados de Concepción. El estudio utilizó un enfoque cuantitativo, de carácter descriptivo, de corte transversal. La muestra fue de 71 escolares, de los cuales 48 pertenecen al Grupo Educación Física y 23 al Grupo Extracurricular. Para la evaluación se utilizó el Test TGMD-2 (Ulrich, 2000). Los resultados mostraron que existen diferencias significativas entre ambos grupos $(\mathrm{p}<0,05)$, observándose un mejor desarrollo motor en los escolares del Grupo Extracurricular.

\section{PALABRAS CLAVE}

Desarrollo motor, talleres deportivos extracurriculares, educación física.

\begin{abstract}
This research sought to determine if there are differences in motor development between 9 and 10 year old children who only participate in their physical education class and those who, in addition to this class, participate in extracurricular sports workshops offered by subsidized private schools in Concepción, Chile. The study used a quantitative, descriptive, cross-sectional approach. The sample was made up of 71 students, of which 48 belong to the Physical Education Group and 23 to the Extracurricular Group. The TGMD-2 Test was used for the evaluation (Ulrich, 2000). The results showed that there are significant differences between both groups $(\mathrm{p}<0.05)$, and where the students from Extracurricular Group showed better motor development.
\end{abstract}

\section{Key words}

Motor development, extracurricular sports workshops, physical education.

* Facultad de Ciencias de la Educación, Pedagogía en Educación Física, Universidad San Sebastián, Concepción, Chile. 


\section{INTRODUCCIÓN}

El Desarrollo Motor, hace referencia al refinamiento y perfeccionamiento a nivel motriz, según Candel (2004), el Movimiento Humano es sensible a la estimulación, más aún si es realizada en los primeros años de vida, y en los ambientes familiares y escolares.

La relevancia y la presencia de la actividad física y el deporte en la vida de la población en edad escolar es actualmente, uno de los fenómenos culturales de mayor preocupación, es por ello que existe la necesidad de que este tipo de actividades formen parte de las actividades escolares desde muy temprana edad, para que a partir de experiencias enriquecedoras y satisfactorias puedan disfrutar de la actividad motriz y generar un hábito de práctica para toda su vida. En ese sentido, a pesar de que cada vez existe mayor evidencia acerca del rol potenciador del movimiento para el desarrollo del ser humano, éste ha decrecido. Es así como Gatica y Vargas (2004) nos señalan que el nivel y patrones de actividad física de grupos poblacionales ha disminuido en las actuales generaciones en relación a las anteriores, con las respectivas repercusiones que esto puede tener sobre el nivel de desarrollo de la aptitud motora, la salud y la calidad de vida, haciendo necesario reflexionar acerca del papel que la actual Educación Física está cumpliendo en el desarrollo de los niños y niñas, y en el logro de sus objetivos de preparar individuos más autónomos, físicamente activos y de pleno desarrollo motor.

Según Ruiz Pérez (1994), El desarrollo en los seres humanos, hace referencia a los cambios en el nivel de funcionamiento de forma general, implica la aparición y ampliación de las capacidades para funcionar en un nivel más complejo. Son variaciones de tipo cualitativo y cuantitativo, que afectan las estructuras y funciones orgánicas que resultan de la interacción del crecimiento, la maduración y la experiencia.

En el caso del Desarrollo de la dimensión Motriz, este proceso se lleva a cabo mediante el progreso desde movimientos simples y desorganizados para paulatinamente ir alcanzando habilidades organizadas y complejas. Tal desarrollo no se produce de forma aislada, más bien se verá influido por las características biológicas que el niño posea (tales como la herencia, el crecimiento y la maduración), y por el ambiente en que se desarrolle, específicamente por las oportunidades de movimiento que se encuentran en su entorno, provistas por la familia, en primer lugar, y luego por la escuela, en las actividades físicas programadas en ella.

Stodden y cols.(2008) señalan que, establecer hábitos de actividad física desde edades tempranas, favorece el desarrollo motor y motivarán a mantenerse físicamente activo a lo largo de la existencia, además determinaron que los niveles de actividad física permiten elevar los rendimientos motores globales, tal como lo afirman Düger y cols (1999) quienes concluyen que el refinamiento de los patrones va a depender de la cantidad de práctica y del tipo de instrucción dada para el mejoramiento del movimiento.

En Chile, de acuerdo al Ministerio de Educación (2013), es en la Educación Inicial, Pre- Escolar y Escolar Básica, donde se debe asegurar que todos los niños reciban la estimulación y educación motriz adecuada a su respectiva edad, así en el caso de la educación básica establece un total de 152 horas anuales (4 horas semanales) de educación física para los establecimientos con jornada escolar completa. Además se señala que se debe pretender, durante estas clases, mejorar las habilidades motrices, fomentar actitudes hacia el juego y el deporte, promoviendo estilos de vida activos y saludables.

Teniendo en cuenta lo anterior, según Mollá (2007) en las clases de educación física es muy difícil conseguir efectos positivos en lo que se refiere a la condición física y salud, ya que el tiempo real de práctica es insuficiente. Por este motivo y otros, en nuestro país, que las Escuelas que cuentan con Jornada Escolar Completa tienen la posibilidad de complementar la educación de sus estudiantes por medio de actividades extracurriculares, fortaleciendo actitudes personales y el desarrollo integral de sus educandos. De esta forma, la actividad 
física-deportiva se puede incentivar a través de las actividades complementarias y extraescolares. En palabras del mismo Mollá (2007) estas actividades son un complemento del currículo del aula, ya sea en horario lectivo o fuera de él, siendo un instrumento más al servicio del profesor, con la finalidad de complementar los aprendizajes de las clases que recibe el estudiante, en un ambiente menos formal, más distendido, con carácter más o menos voluntario.

De esta forma, las actividades deportivas durante la edad escolar, requieren, de acuerdo a Garcia y cols. (2000), de un tratamiento pedagógico, fundamental para el desarrollo adecuado de las habilidades motrices, y de estrategias didácticas basadas en juegos, recomendadas como elemento educativo de vital importancia; en esta línea, de acuerdo a Blásquez (1995), las situaciones motrices más apropiadas, serían los juegos simplificados y de reglas flexibles, adaptados a la estructura particular de los deportes que se comienzan a aprender y a la población infantil que los va a ejecutar.

Unido a lo anterior, es de especial relevancia, en palabras de Siedentop (1998) la utilización de estrategias de enseñanza activa y aprendizaje cooperativo, así como la entrega oportuna de feedbacks y evaluaciones por parte del docente, que sumado a las interacciones entre los participantes, favorecerán el desarrollo y la adquisición de los diferentes aprendizajes.

De esta forma la Iniciación Deportiva, tendrá un marcado acento pedagógico, procurando, de acuerdo a Sanchez Bañuelos (1986) y Blázquez (1995), el desarrollo de aprendizajes genéricos básicos, de socialización y de operatividad motriz, fortaleciendo el sentido lúdico deportivo, por sobre las exigencias competitivas.

A partir de los antecedentes antes mencionados, se plantea el objetivo de determinar si existen diferencias en el desarrollo motor en escolares de 9 y 10 años, que participan únicamente de la clase de educación física, y aquellos que además de la clase, participan de talleres deportivos extracurriculares pertenecientes a colegios particulares subvencionados de Concepción.

\section{MÉTODOS}

Se llevó a cabo un estudio de enfoque cuantitativo de alcance descriptivo, con un diseño no-experimental, transversal. A través de un muestreo No probabilístico, se seleccionó a 71 escolares pertenecientes a dos establecimientos particulares subvencionados de Concepción, de los cuales $\mathrm{N}=23$ pertenecen al Grupo de escolares que asisten regularmente a actividades deportivas-extracurriculares (en adelante Grupo Extracurricular $=$ GEX) y a 48 a escolares que participan exclusivamente de la clases de Educación Fisica (en adelante Grupo Educación Física $=\mathrm{GEF})$.

Criterios de Inclusión: 1) Pertenecer al $4^{\circ}$ básico de los establecimientos seleccionados; 2) Tener entre 9 y 10 años de edad; 3 ) a la fecha de las evaluaciones haber Asistido regularmente a las clases de Educación Física, 80\% de asistencia para ambos grupos; 4) En el caso del GEX, además, asistir al menos al $80 \%$ de actividades deportivas extracurriculares realizadas a la fecha de la evaluación. 5) No sufrir algún tipo de discapacidad física, mental u otra, que afecte el resultado de las evaluaciones; 6) presentación del consentimiento informado por parte del apoderado.

Como procedimiento se seleccionaron establecimientos educacionales particulares subvencionados de Concepción, que contaran con actividades deportivas extracurriculares programadas, ejecutadas sistemáticamente $\mathrm{y}$ a cargo de Profesores de Educación Física. Se les envió una carta a los directivos declarando el objetivo de la investigación e invitándolos a participar, lo que se formalizó a través de la firma de dicha solicitud. Luego de esto, se les envió un consentimiento informado a los apoderados de los escolares de entre 9 años y 10 años 11 meses de edad de cada establecimiento, con el fin de informar respecto del objetivo del estudio, el carácter anónimo y voluntario de la participación, y que las evaluaciones no revestían ningún perjuicio para la salud de los escolares; el consentimiento debía ser firmado por los apoderados autorizando la participación de su pupilo. 
Para la evaluación se utilizó el test de Desarrollo Motor Grueso de Ulrich (2000) TGMD2. Este test consta de dos subpruebas que mide en cifras las habilidades motoras gruesas que se desarrollan temprano en la vida. Fue diseñado para evaluar el funcionamiento motor en cifras. Fue validado por Gatica -Vargas (2002) para ser utilizado en Chile.

El objetivo del TGMD-2, es la medición de las habilidades motoras gruesas que se desarrollan en la infancia. La prueba mide 12 habilidades motoras gruesas observadas en los niños de pre-escolar y primaria.

El objetivo principal de esta prueba es Identificar a los niños que en el desarrollo de las habilidades motoras gruesas presentan niveles de ejecución bajos o superiores al que deberían poseer respecto a la edad en que se encuentran.

Las dos sub-pruebas que conforman el TGMD-2 evalúan diferentes aspectos del desarrollo motor grueso: locomoción y control de objetos.

\section{RESULTADOS}

A continuación se presentan los resultados del Test de desarrollo motor grueso TGMD-2 (Ulrich, 2000), se divide en los test locomotor y de control de objetos. Para el análisis descriptivo de los datos se utilizaron medidas de tendencia central como Media, Moda y Mediana, además de medidas de variabilidad como Rango, Puntaje Mínimo, Puntaje Máximo y Desvío Estándar. Para la realización de las tablas y figuras se utilizó el programa computacional Microsoft Office Excel 2013, para Windows 10 home single language

Para el análisis correlacional de los datos, con el fin de determinar las diferencias entre las medias de las evaluaciones de los Grupos Evaluados se utilizó la prueba " $t$ " de Student, del programa estadístico SPSS 20.0. Junto con ello, se realizó la prueba de "KolmogorovSmirnov", para verificar la normalidad en la diferencia de puntuación del conjunto de datos por cada grupo, resultando ambas muestras de distribución normal GEX ( $\mathrm{p}=0.952)$, GEF $(\mathrm{p}=0.679)$.

Tabla 1

Análisis descriptivo de resultados: sub test locomotor (TGMD-2).

\begin{tabular}{ccccccccc}
\hline Grupo & N & Mínimo & Máximo & Rango & Moda & Mediana & Media & Desv. Estándar \\
\hline GEF & 48 & 27 & 48 & 21 & 39 & 36,5 & 37,1 & 4,5 \\
\hline GEX & 23 & 35 & 48 & 13 & 46 & 44 & 43,3 & 3,4 \\
\hline
\end{tabular}

Tabla 1. Permite apreciar mejores resultados en el GEX respecto al GEF, la media, moda, mediana y el puntaje mínimo son mayores, por su parte tanto el rango como la desviación estándar son menores en el GEX. De esta forma el GEX se presenta como un grupo más homogéneo y de mejores resultados que el GEF.

Tabla 2

Análisis descriptivo de resultados: sub test control de objetos (TGMD-2).

\begin{tabular}{ccccccccc}
\hline Grupo & N & Mínimo & Máximo & Rango & Mediana & Moda & Media & Desv. Estándar \\
\hline GEF & 48 & 25 & 48 & 23 & 36 & 36 & 35,8 & 4,0 \\
\hline GEX & 23 & 34 & 48 & 14 & 42 & 41 & 41,8 & 3,9 \\
\hline
\end{tabular}

Tabla 2. Muestra que el GEX, tiene mejores resultados tanto en la media, moda, mediana y puntaje mínimo, además de un rango más acotado, siendo así el GEX más homogéneo y con mejores puntajes que el GEF. 
Tabla 3

Análisis descriptivo de resultados: Puntaje General Test TGMD-2.

\begin{tabular}{ccccccccc}
\hline Grupo & N & Mínimo & Máximo & Rango & Mediana & Moda & Media & Desv. Estándar \\
\hline GEF & 48 & 55 & 109 & 54 & 76 & 76 & 73,0 & 6,3 \\
\hline GEX & 23 & 85 & 121 & 36 & 94 & 97 & 85,1 & 5,0 \\
\hline
\end{tabular}

Tabla 3. Permite observar un mejor puntaje en general en los resultados del GEX, junto con ello tanto el rango como el desvío estándar son menores. Por lo tanto, el GEX obtiene mejores resultados y se presenta como un grupo más equilibrado en sus resultados que el GEF.

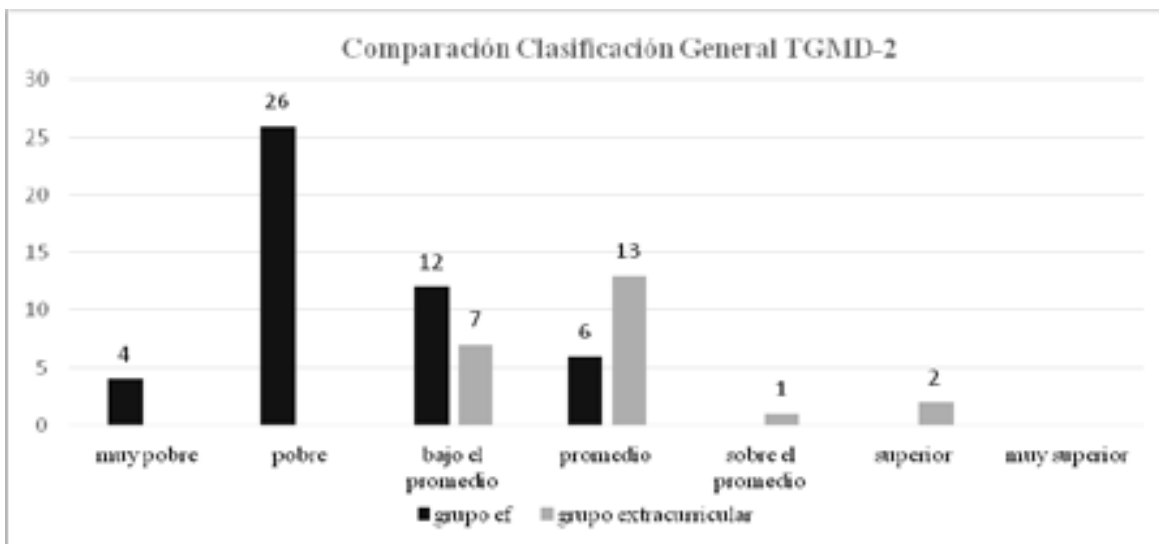

Figura 1. Clasificación General de Resultados TGMD-2.

Figura 1. Permite apreciar un déficit en los resultados del GEF, quienes se ubican mayormente en las categorías Pobre $(\mathrm{N}=26)$ y Bajo el promedio $(\mathrm{N}=12)$. En cambio, los evaluados del GEX están preferentemente en las categorías Promedio $(\mathrm{N}=13)$. Además, ningún escolar del GEF, se encuentra en las categorías "sobre el promedio" o "superior", en cambio, si existen evaluados en dichas categorías de la evaluación del GEX $(\mathrm{N}=3)$. A partir de lo señalado, se observan, en general, mejores resultados en el GEX, ya que los puntajes obtenidos los ubican, en mayor número, en mejores categorías de evaluación respecto al GEF.

\section{Tabla 4}

Análisis diferencia de medias: $T$ de Student.

\begin{tabular}{|c|c|c|c|c|c|c|c|c|c|c|}
\hline \multicolumn{11}{|c|}{ Prueba de muestras independientes } \\
\hline & & \multicolumn{2}{|c|}{$\begin{array}{c}\text { Prueba de Levene } \\
\text { para la igualdad } \\
\text { de varianzas }\end{array}$} & \multicolumn{7}{|c|}{ Prueba T para la igualdad de medias } \\
\hline & & \multirow[t]{2}{*}{$\mathrm{F}$} & \multirow[t]{2}{*}{ Sig. } & \multirow[t]{2}{*}{$\mathrm{t}$} & \multirow[t]{2}{*}{$\mathrm{gl}$} & \multirow[t]{2}{*}{$\begin{array}{c}\text { Sig. } \\
\text { (bilateral) }\end{array}$} & \multirow[t]{2}{*}{$\begin{array}{l}\text { Diferencia } \\
\text { de medias }\end{array}$} & \multirow[t]{2}{*}{$\begin{array}{l}\text { Error típ. de } \\
\text { la diferencia }\end{array}$} & \multicolumn{2}{|c|}{$\begin{array}{c}95 \% \text { intervalo de } \\
\text { confianza para la } \\
\text { diferencia }\end{array}$} \\
\hline & & & & & & & & & Inferior & Superior \\
\hline $\begin{array}{c}\text { Puntaje } \\
\text { test }\end{array}$ & $\begin{array}{l}\text { Se han } \\
\text { asumido } \\
\text { varianzas } \\
\text { iguales }\end{array}$ & 0.171 & 0.68 & -7.969 & 71 & 0.001 & -12.111 & 1.52 & -15.143 & -9.08 \\
\hline
\end{tabular}


Tabla 4. Al realizar la prueba T-Student en la diferencia de puntación entre los resultados obtenidos en el TGMD-2 del GEF y GEX, se observa un valor $\mathrm{p}<0.05$, por lo tanto, los promedios de los puntajes son diferentes entre ambos grupos, confirmando que sí existen diferencias significativas en los resultados obtenidos en las evaluaciones realizadas.

\section{DISCUSIÓN}

$\mathrm{Al}$ analizar los resultados en la clasificación general del TGMD-2, se observa que el $89 \%$ de los escolares del GEF obtienen puntajes que los ubican en rangos menores de desarrollo motor de acuerdo a su edad cronológica, lo que coincide con los resultados obtenidos por otras investigaciones en Chile de similares características, que además utilizaron el mismo test, Gatica y Vargas (2004 y 2006), Luarte y cols. (2014), Luarte, Poblete y Flores (2014), Poblete, Flores y Bustos (2013), Poblete, Morilla y Quintana (2015), Luna y cols. (2016), lo que deja en evidencia, por los datos obtenidos en dichos estudios y en el presente, que la estimulación que están recibiendo los escolares pareciera ser insuficiente a los requerimientos de su dimensión motriz.

Por su parte, los resultados en la clasificación general del TGMD-2 para el GEX, muestra que el $69 \%$ de los evaluados evidenciaron resultados motores esperados para su edad, lo que coincide con resultados obtenidos por Poblete, Flores y Bustos (2013), Leiva y cols. (2015), quienes observaron que los escolares que reciben estimulación deportiva extracurricular durante la niñez obtienen un nivel de desarrollo motor adecuado para la edad; de esta forma se validan los planteamientos de Ruiz Pérez (1994), Gallahue y Ozmun (2006) quienes resaltan la relevancia del medio ambiente y de la estimulación para el perfeccionamiento de las Habilidades Motrices Básicas, sobre todo en edades tempranas.

Al comparar los resultados obtenidos en las evaluaciones en el desarrollo motor, el GEX, obtiene en promedio una diferencia significativa $(\mathrm{p}<0,05)$ respeto al GEF, de esta forma los resultados obtenidos son consistentes con los resultados de Fritz y Vargas (2013), y con lo señalado por Blásquez (1995), Garcia y cols. (2000) y Mollá (2007), quienes concuerdan en que las actividades deportivas extra curriculares con tratamiento pedagógico, permiten fortalecer los aprendizajes motores, en especial si se producen en un ambiente lúdico, con un programación coherente, estructurada y acorde a la edad de los participantes, soslayando así los déficits motores producto de la creciente inactividad física en la población infantil. Junto con ello, los escolares pueden acceder a mayor niveles de actividad física, tal cual lo indica la Organización Mundial de la Salud (2010), que recomienda, al menos 60 minutos de actividad física diaria a los jóvenes hasta los 17 años, además, si estas son vigorosas, como las actividades deportivas, permitirán fortalecer tejidos musculares, óseos y articulares.

Al observar los resultados obtenidos tanto en el sub test Locomotor y sub test control de objetos, el GEF muestra sus mejores resultados en sub test Control de Objetos, donde el $23 \%$ de los evaluados se ubica en las categorías "Promedio" y "sobre el Promedio", por su parte el GEX, muestra, mejores y más homogéneos resultados en ambos sub test en comparación al GEF, ya que en el sub test Locomotor un $69 \%$ de los escolares y en el Control de Objetos el $61 \%$ de ellos, obtienen puntajes que los ubican en las categorías "Promedio", "sobre el Promedio" y "Superior", lo que es consistente con los hallazgos de Poblete, Flores y Bustos (2013) Fritz y Vargas (2013), Luna y cols. (2016), los que concluyen que un mayor tiempo de práctica motriz permite obtener mejores resultados en los test de desarrollo motor. Además es coincidente con los planteamientos de Düger y cols (1999), Stodden y cols. (2008) quienes señalan que aumentar los niveles de actividad física permite elevar los rendimientos motores globales, lo que involucra el mejoramiento en habilidades motrices locomotoras, equilibratorias y de control de objetos. 


\section{CONCLUSIÓN}

Al determinar si existen diferencias en el desarrollo motor en escolares de 9 y 10 años, que participan únicamente de la clase de educación física, y aquellos que además de la clase, participan de talleres deportivos extracurriculares pertenecientes a colegios particulares subvencionados de Concepción, los resultados muestran que los escolares que participan de la clase de educación física y además participan de los de talleres deportivos extracurriculares, presentan un mejor desarrollo motor que aquellos que solo asisten a la clase de Educación Física. Prueba de ello es que los resultados del GEX, fueron superiores al GEF, en los subtest locomotor y control de objetos, además en la clasificación general del TGMD-2 se ubicaron en categorías superiores de clasificación con respecto al GEF, por su parte, las diferencias de los promedios de estos grupos fue significativa $(\mathrm{p}<0,05)$.

Pese a que los resultados indican que el GEX obtiene un desarrollo motor significativamente mejor que el GEF, aún no se cuenta con evidencia empírica suficiente para atribuir esta diferencia a la exclusiva participación en las actividades deportivas extracurriculares, debido al reducido tamaño de la muestra y a la ausencia de control-medición de otras variables influyentes, como la maduración, estimulación recibida en otros contextos y estilos de crianza.

\section{REFERENCIAS BIBLIOGRÁFICAS}

Blázquez, D. (1995). La iniciación deportiva y el deporte escolar. Barcelona: INDE.

Candel, I. (2004). Prevención desde el ámbito educativo: Patologías no evidentes. En J. Pérez-López y A.G. Brito de la Nuez (Eds.), Manual de Atención Temprana (119-132). Madrid: Pirámide.

Düger, T., Bumin, G., Uyanik, M., Aki, E., \& Kayihan, H. (1999).The assesment of Bruininks-Oseretsky test of motor proficiency in children. Pediatric Rehabilitation. Vol. 3 (3) 125-131.
Fritz, J. \& Vargas, R. (2013). Impacto de actividades lúdicas no competitivas sobre las habilidades motoras gruesas en niños y niñas pre escolares. Revista Ciencias de la Actividad Física UCM. Vol. 14 (1) 31-37.

Gallahue, D. \& Ozmun, J.C. (2006). Understanding Motor Development: Infants, Children, Adolescents, and Adults. (6ed.). Boston. MA: McGraw-Hill.

García, A. Gutiérrez, F. Marqués, J. Román, R. Ruiz, F. Samper M. (2000). Los Juegos en la Educación Física de los 6 a los 12 años. Barcelona: INDE.

Gatica, P., Vargas, R. y cols (2002). Evaluación del desarrollo motor en escolares de 6 a 10 años de la región del Maule. Talca: UCM-IND.

Gatica, P.; Vargas, R. (2004). Evaluación del desarrollo motor de los escolares de 6 a 10 años en la región del Maule Talca. Revista Ciencias de la Actividad Física. Vol. 7 (1) 67-79.

Gatica, P.; Vargas, R. (2006). Actividades de estimulación motriz para el desarrollo de habilidades motoras básicas en el subsector de Educación Física de NB1. Revista Ciencias de la Actividad Física UCM. Vol. 8 (1) 7-24.

Leiva, M., Alvarado, C., Gallardo, R., Vargas, R., \& Martínez, C. \&. (2015). Desarrollo motor en escolares con diferentes aprestos formativos motrices. Revista Ciencias de la Actividad Física UCM. Vol. 16 (1), 19-28.

Luarte, C.; Poblete, F. y Flores, C. (2014). Nivel de desarrollo motor grueso en preescolares sin intervención de profesores de Educación Física, Concepción, Chile. Revista Ciencias de la Actividad Física UCM. Vol. 15 (1)7-16. 
Luarte, C., Rodrigues, R., Luna, P., Gutierrez, C. y Carreño, M. (2014). Desarrollo motor Grueso: Efectos de un programa de estimulación motriz, basado en juegos motores, para escolares con déficit motor del nb1, en un colegio particular de la ciudad de Concepción, Chile. Conexões: Revista da Faculdade de Educação Física da UNICAMP. Vol. 12 (1) 85-106.

Luna, P., Aravena, J., Contreras, D., Fabres, C., \& Faúndez, F. (2016). Efectos en el desarrollo motor de un programa de estimulación de habilidades motrices básicas en escolares de $5^{\circ}$ año Básico de colegios particulares subvencionados de Concepción. Revista Ciencias de la Actividad Física UCM. Vol. 17 (1) 2736.

Ministerio de Educación, República de Chile (2013). Programa de Estudio para Cuarto Año Básico. Unidad de Currículum y Evaluación. Extraído de: http://www.curriculumenlineamineduc.cl/605/articles-20741_programa. pdf. Recuperado el 10-03-2017.

Mollá, M. (2007). La influencia de las actividades Extraescolares en los Hábitos deportivos de los Escolares. Revista Internacional de Medicina y Ciencias de la Actividad Física y el Deporte. Vol. 7 (27), 241-252.

Organización Mundial de la Salud (2010). Recomendaciones mundiales sobre actividad física para la salud. Ginebra: Organización Mundial de la Salud.

Poblete, F., Flores, C. y Bustos S.(2013). Desarrollo Motor grueso en alumnos de 8, 9 y 10 años de edad en clases de educación física y talleres extracurriculares. Revista de Ciencias de la Actividad Física UCM. Vol. 14 (2), 21-30.
Poblete, F., Morilla, C., Quintana, C. (2015). Nivel de desarrollo motor grueso en pre-escolares sin intervención de profesores de Educación Física, Valdivia. Horizonte: Ciencias de la Actividad Física. Vol. 6 (2) 33-40.

Ruiz Pérez, L.M. (1994). Desarrollo Motor y Actividades Físicas. Madrid: Gimnos.

Sánchez Bañuelos, F. (1986). Bases para una didáctica de la educación física y el deporte. Madrid: Gymnos.

Siedentop D. (1998). Aprender a Enseñar la Educación Física. Barcelona: INDE.

Stodden, D., Goodway, J., Langendorter, S., Roberton, M., Rudisill, M., Garcia, C., et al. (2008). A developmental Perspective on the role of Motor Skill Competence in Physical Activity: An Emergent Relationship. Vol. 60 (2) 290-306.

Ulrich, D.A. (2000). The test of gross motor development (2nd Ed.). Austin, TX: PROED Publishers.

\section{Dirección para correspondencia}

Pablo Luna

Profesor de educación física

Magíster en educación Física y deporte.

Universidad San Sebastián Concepción

Contacto:

pablo.luna@uss.cl

Recibido: 04-06-2017

Aceptado: 11-07-2017 